\title{
Exact Traveling Wave Solutions for Wick-Type Stochastic Schamel KdV Equation
}

\author{
Hossam A. Ghany ${ }^{1,2}$ and M. Zakarya ${ }^{3}$ \\ ${ }^{1}$ Department of Mathematics, Faculty of Science, Taif University, Al Hawiyah, Taif 888, Saudi Arabia \\ ${ }^{2}$ Department of Mathematics, Helwan University, Cairo, Egypt \\ ${ }^{3}$ Department of Mathematics, Faculty of Science, Al-Azhar University, Assiut 71524, Egypt
}

Correspondence should be addressed to Hossam A. Ghany; h.abdelghany@yahoo.com

Received 29 May 2014; Accepted 9 October 2014; Published 15 December 2014

Academic Editor: Alkesh Punjabi

Copyright (C) 2014 H. A. Ghany and M. Zakarya. This is an open access article distributed under the Creative Commons Attribution License, which permits unrestricted use, distribution, and reproduction in any medium, provided the original work is properly cited.

F-expansion method is proposed to seek exact solutions of nonlinear partial differential equations. By means of Hermite transform, inverse Hermite transform, and white noise analysis, the variable coefficients and Wick-type stochastic Schamel KdV equations are completely described. Abundant exact traveling wave solutions for variable coefficients Schamel KdV equations are given. These solutions include exact stochastic Jacobi elliptic functions, trigonometric functions, and hyperbolic functions solutions.

\section{Introduction}

In this paper, we investigate the variable coefficients Schamel $\mathrm{KdV}$ equations $[1,2]$ :

$$
\begin{array}{r}
u_{t}+\left[g_{1}(t) u^{1 / 2}+g_{2}(t) u\right] u_{x}+g_{3}(t) u_{x x x}=0, \\
(t, x) \in \mathbb{R}_{+} \times \mathbb{R},
\end{array}
$$

where $g_{1}(t), g_{2}(t)$, and $g_{3}(t)$ are bounded measurable or integrable functions on $\mathbb{R}_{+}$. Random wave is an important subject of stochastic partial differential equations (SPDEs). Many authors have studied this subject. Wadati first introduced and studied the stochastic KdV equations and gave the diffusion of soliton for KdV equation under Gaussian noise in [3, 4] and others [5-9] also researched stochastic KdV equations. Xie first introduced Wick-type stochastic KdV equations on white noise space and showed the auto-Backlund transformation and the exact white noise functional solutions in [10]. Furthermore, Xie [11-14] and Ghany et al. [15-21] researched some Wick-type stochastic wave equations using white noise analysis.

In this paper we use F-expansion method for finding new periodic wave solutions of nonlinear evolution equations in mathematical physics, and we obtain some new periodic wave solutions for Schamel KdV equations. This method is more powerful and will be used in further works to establish more entirely new solutions for other kinds of nonlinear partial differential equations arising in mathematical physics. The effort in finding exact solutions to nonlinear equations is important for the understanding of most nonlinear physical phenomena, for instance, the nonlinear wave phenomena observed in the fluid dynamics, plasma, and optical fibers $[1,2]$. Many effective methods have been presented such as homotopy analysis method [22], variational iteration method [23, 24], tanh-function method [25-27], homotopy perturbation method [28-30], tanh-coth method [26, 31, 32], Exp-function method [33-38], Jacobi elliptic function expansion method [39-42], and F-expansion method [4346]. The main objective of this paper is using F-expansion method to construct the exact traveling wave solutions for Wick-type stochastic Schamel KdV equations via the Wicktype product, Hermite transform, and white noise analysis. If (1) is considered in a random environment, we can get stochastic Schamel KdV equations. In order to give the exact solutions of stochastic Schamel KdV equations, we only consider this problem in white noise environment. 
We will study the following Wick-type stochastic Schamel $\mathrm{KdV}$ equations:

$$
\begin{aligned}
U_{t}+ & {\left[G_{1}(t) \diamond U^{\diamond 1 / 2}+G_{2}(t) \diamond U\right] \diamond U_{x} } \\
+ & G_{3}(t) \diamond U_{x x x}=0,
\end{aligned}
$$

where " $\diamond$ " is the Wick product on the Kondratiev distribution space $(\mathcal{S})_{-1}$ and $G_{1}(t), G_{2}(t)$, and $G_{3}(t)$ are $(\mathcal{S})_{-1}$ valued functions [47].

\section{Description of the F-Expansion Method}

In order to simultaneously obtain more periodic wave solutions expressed by various Jacobi elliptic functions to nonlinear wave equations, we introduce an F-expansion method which can be thought of as a succinctly overall generalization of Jacobi elliptic function expansion. We briefly show what Fexpansion method is and how to use it to obtain various periodic wave solutions to nonlinear wave equations. Suppose a nonlinear wave equation for $u(t, x)$ is given by

$$
\Psi_{1}\left(u, u_{t}, u_{x}, u_{x x}, u_{x x x}, \ldots\right)=0,
$$

where $u=u(t, x)$ is an unknown function and $\Psi_{1}$ is a polynomial in $u$ and its various partial derivatives in which the highest order derivatives and nonlinear terms are involved. In the following we give the main steps of a deformation F-expansion method.

Step 1. Look for traveling wave solution of (3) by taking

$$
u(t, x)=u(\xi), \quad \xi(t, x)=k x+\int_{0}^{t} \theta(\tau) d \tau+c .
$$

Hence, under the transformation in (4), then, (3) can be transformed into ordinary differential equation (ODE) as follows:

$$
\Psi_{2}\left(u, \theta u^{\prime}, k u^{\prime}, k^{2} u^{\prime \prime}, k^{3} u^{\prime \prime \prime}, \ldots\right)=0 .
$$

Step 2. Suppose that $u(\xi)$ can be expressed by a finite power series of $F(\xi)$ of the form

$$
u(t, x)=u(\xi)=\sum_{i=0}^{N} a_{i} F^{i}(\xi),
$$

where $a_{0}, a_{1}, \ldots, a_{N}$ are constants to be determined later, while $F^{\prime}(\xi)$ in (6) satisfies

$$
\left[F^{\prime}(\xi)\right]^{2}=P F^{4}(\xi)+Q F^{2}(\xi)+R
$$

and hence holds for $F(\xi)$ :

$$
\begin{gathered}
F^{\prime} F^{\prime \prime}=2 P F^{3} F^{\prime}+Q F F^{\prime}, \\
F^{\prime \prime}=2 P F^{3}+Q F, \\
F^{\prime \prime \prime}=6 P F^{2} F^{\prime}+Q F^{\prime},
\end{gathered}
$$

where $P, Q$, and $R$ are constants.
Step 3. The positive integer $N$ can be determined by considering the homogeneous balance between the highest derivative term and the nonlinear terms appearing in (5). Therefore, we can get the value of $N$ in (6).

Step 4. Substituting (6) into (5) with condition (7), we obtain polynomial in $F^{i}(\xi)\left[F^{\prime}(\xi)\right]^{j}(i=0 \pm 1, \pm 2, \ldots, j=0,1)$. Setting each coefficient of this polynomial to be zero yields a set of algebraic equations for $a_{0}, a_{1}, \ldots, a_{N}$ and $\theta$.

Step 5. Solving the algebraic equations with the aid of Maple we have $a_{0}, a_{1}, \ldots, a_{N}$ and $\theta$ can be expressed by $P, Q$, and $R$. Substituting these results into F-expansion (6), then a general form of traveling wave solution of (3) can be obtained.

Step 6. Since the general solutions of (6) have been well known for us, choose properly $P, Q$, and $R$ in ODE (7) such that the corresponding solution $F(\xi)$ of it is one of Jacobi elliptic functions (see Appendices A, B, and C) [43-45].

\section{Exact Traveling Wave Solutions of (2)}

In this section, we apply Hermite transform, white noise theory, and F-expansion method to explore soliton and periodic wave solutions for (2). Applying Hermite transform to (2), we get the deterministic equation

$$
\begin{gathered}
\widetilde{U}_{t}(t, x, z)+\left\{\widetilde{G}_{1}(t, z) \widetilde{U}^{1 / 2}(t, x, z)\right. \\
\left.+\widetilde{G}_{2}(t, z) \widetilde{U}(t, x, z)\right\} \widetilde{U}_{x}(t, x, z) \\
+\widetilde{G}_{3}(t, z) \widetilde{U}_{x x x}(t, x, z)=0,
\end{gathered}
$$

where $z=\left(z_{1}, z_{2}, \ldots\right) \in\left(\mathbb{C}^{\mathbb{N}}\right)_{c}$ is a vector parameter. To look for the traveling wave solution of (3), we make the transformations $\widetilde{G}_{1}(t, z):=g_{1}(t, z), \widetilde{G}_{2}(t, z):=g_{2}(t, z)$, $\widetilde{G}_{3}(t, z):=g_{3}(t, z)$, and $\widetilde{U}(t, x, z)=: u(t, x, z), u=v^{2}$, $v(t, x, z)=V(\xi)$, with

$$
\xi(t, x, z)=k\left[x-\int_{0}^{t} \theta(\tau, z) d \tau\right]+c,
$$

where $k$ and $c$ are arbitrary constants which satisfy $k \neq 0$ and $\theta(t, z)$ is a nonzero function of the indicated variables to be determined later. Thus, (3) can be transformed into the following ODE:

$$
-\theta V V^{\prime}+\left[g_{1} V^{2}+g_{2} V^{3}\right] V^{\prime}+g_{3} k^{2}\left[V V^{\prime \prime \prime}+3 V^{\prime} V^{\prime \prime}\right]=0,
$$

where $V^{\prime}=d V / d \xi$. The balancing procedure implies that $N=1$. Therefore, in view of F-expansion method the solution of (3) can be expressed in the form

$$
V(t, x, z)=V(\xi)=a_{0}+a_{1} F(\xi(t, x, z)),
$$

where $a_{0}, a_{1}$ are constants to be determined later. Substitute (12) with conditions (7) and (8) into (11) and collect all terms 
with the same power of $F^{i}(\xi)\left[F^{\prime}(\xi)\right]^{j}(i=0, \pm 1, \pm 2, \ldots, j=$ $0,1)$ as follows:

$$
\begin{aligned}
& {\left[-\theta a_{0} a_{1}+g_{1} a_{0}^{2} a_{1}+g_{2} a_{0}^{3} a_{1}+g_{3} k^{2} a_{0} a_{1} Q\right] F^{\prime}} \\
& +\left[-\theta a_{1}^{2}+2 g_{1} a_{0} a_{1}^{2}+3 g_{2} a_{0}^{2} a_{1}^{2}+4 g_{3} k^{2} a_{1}^{2} Q\right] F F^{\prime} \\
& \quad+\left[g_{1} a_{1}^{3}+3 g_{2} a_{0} a_{1}^{3}+6 g_{3} k^{2} a_{0} a_{1} P\right] F^{2} F^{\prime} \\
& \quad+\left[g_{2} a_{1}^{4}+12 g_{3} k^{2} a_{1}^{2} P\right] F^{3} F^{\prime}=0 .
\end{aligned}
$$

Setting each coefficient of $F^{i}(\xi)\left[F^{\prime}(\xi)\right]^{j}$ to be zero, we get a system of algebraic equations which can be expressed by

$$
\begin{gathered}
\left(-\theta+g_{1} a_{0}+g_{2} a_{0}^{2}+g_{3} k^{2} Q\right) a_{0} a_{1}=0, \\
\left(-\theta+2 g_{1} a_{0}+3 g_{2} a_{0}^{2}+4 g_{3} k^{2} Q\right) a_{1}^{2}=0, \\
\left(g_{1} a_{1}^{2}+3 g_{2} a_{0} a_{1}^{2}+6 g_{3} k^{2} a_{0} P\right) a_{1}=0, \\
\left(g_{2} a_{1}^{2}+12 g_{3} k^{2} P\right) a_{1}^{2}=0,
\end{gathered}
$$

with solving the above system to get the following coefficients:

$$
\begin{gathered}
a_{1}= \pm \sqrt{\frac{-12 k^{2} g_{3}(t, z) P}{g_{2}(t, z)}}, \\
a_{0}=-\frac{2 g_{1}(t, z)}{5 g_{2}(t, z)}, \\
\theta=\frac{-6 g_{1}^{2}(t, z)+25 k^{2} g_{2}(t, z) g_{3}(t, z) Q}{25 g_{2}(t, z)} .
\end{gathered}
$$

Substituting coefficient (15) into (12) yields general form solutions to (2):

$$
u(t, x, z)=\left[-\frac{2 g_{1}(t, z)}{5 g_{2}(t, z)} \pm i k \sqrt{\frac{12 g_{3}(t, z) P}{g_{2}(t, z)}} F(\xi(t, x, z))\right]^{2}
$$

with

$$
\begin{aligned}
& \xi(t, x, z) \\
& =k\left\{x-\int_{0}^{t}\left[\frac{-6 g_{1}^{2}(\tau, z)+25 k^{2} g_{2}(\tau, z) g_{3}(\tau, z) Q}{25 g_{2}(\tau, z)}\right] d \tau\right\} \\
& \quad+c .
\end{aligned}
$$

From Appendix A, we give the special cases as follows.

Case 1. If we take $P=1, Q=\left(2-m^{2}\right)$, and $R=\left(1-m^{2}\right)$, then $F(\xi) \rightarrow \operatorname{cs}(\xi)$

$$
\begin{aligned}
& u_{1}(t, x, z) \\
& \quad=\left[-\frac{2 g_{1}(t, z)}{5 g_{2}(t, z)} \pm i k \sqrt{\frac{12 g_{3}(t, z)}{g_{2}(t, z)}} \operatorname{cs}\left(\xi_{1}(t, x, z)\right)\right]^{2},
\end{aligned}
$$

with

$$
\begin{aligned}
& \xi_{1}(t, x, z) \\
& =k\left\{x-\int_{0}^{t}\left[\frac{-6 g_{1}^{2}(\tau, z)+25 k^{2} g_{2}(\tau, z) g_{3}(\tau, z)\left(2-m^{2}\right)}{25 g_{2}(\tau, z)}\right] d \tau\right\} \\
& \quad+c .
\end{aligned}
$$

In the limit case when $m \rightarrow o$, we have $\operatorname{cs}(\xi) \rightarrow \cot (\xi)$; thus (18) becomes

$$
\begin{aligned}
& u_{2}(t, x, z) \\
& \quad=\left[-\frac{2 g_{1}(t, z)}{5 g_{2}(t, z)} \pm i k \sqrt{\frac{12 g_{3}(t, z)}{g_{2}(t, z)}} \cot \left(\xi_{2}(t, x, z)\right)\right]^{2},
\end{aligned}
$$

with

$$
\begin{aligned}
& \xi_{2}(t, x, z) \\
& =k\left\{x-\int_{0}^{t}\left[\frac{-6 g_{1}^{2}(\tau, z)+50 k^{2} g_{2}(\tau, z) g_{3}(\tau, z)}{25 g_{2}(\tau, z)}\right] d \tau\right\} \\
& \quad+c .
\end{aligned}
$$

In the limit case when $m \rightarrow 1$, we have $\operatorname{cs}(\xi) \rightarrow \operatorname{csch}(\xi)$; thus (18) becomes

$$
\begin{aligned}
& u_{3}(t, x, z) \\
& \quad=\left[-\frac{2 g_{1}(t, z)}{5 g_{2}(t, z)} \pm i k \sqrt{\frac{12 g_{3}(t, z)}{g_{2}(t, z)}} \operatorname{csch}\left(\xi_{3}(t, x, z)\right)\right]^{2},
\end{aligned}
$$

with

$$
\begin{aligned}
& \xi_{3}(t, x, z) \\
& =k\left\{x-\int_{0}^{t}\left[\frac{-6 g_{1}^{2}(\tau, z)+25 k^{2} g_{2}(\tau, z) g_{3}(\tau, z)}{25 g_{2}(\tau, z)}\right] d \tau\right\} \\
& \quad+c .
\end{aligned}
$$

Case 2. If we take $P=1 / 4, Q=\left(m^{2}+1\right) / 2$, and $R=(1-$ $\left.m^{2}\right)^{2} / 4$, then $F(\xi) \rightarrow \operatorname{sn} \xi /(\operatorname{cn} \xi \pm \operatorname{dn} \xi)$ and

$$
\begin{aligned}
& u_{4}(t, x, z) \\
& =\left[-\frac{2 g_{1}(t, z)}{5 g_{2}(t, z)} \pm i k \sqrt{\frac{3 g_{3}(t, z)}{g_{2}(t, z)}}\right. \\
& \left.\quad \times\left\{\frac{\operatorname{sn}\left(\xi_{4}(t, x, z)\right)}{\operatorname{cn}\left(\xi_{4}(t, x, z)\right) \pm \operatorname{dn}\left(\xi_{4}(t, x, z)\right)}\right\}\right]^{2},
\end{aligned}
$$


with

$$
\begin{aligned}
& \xi_{4}(t, x, z) \\
& =k\left\{x-\int_{0}^{t}\left[\frac{-12 g_{1}^{2}(\tau, z)+25 k^{2} g_{2}(\tau, z) g_{3}(\tau, z)\left(m^{2}+1\right)}{50 g_{2}(\tau, z)}\right] d \tau\right\} \\
& \quad+c .
\end{aligned}
$$

In the limit case when $m \rightarrow o$, we have sn $\xi /(\operatorname{cn} \xi \pm \operatorname{dn} \xi) \rightarrow$ $\sin \xi /(\cos \xi \pm 1)$; thus (24) becomes

$$
\begin{aligned}
& u_{5}(t, x, z) \\
& \quad=\left[-\frac{2 g_{1}(t, z)}{5 g_{2}(t, z)} \pm i k \sqrt{\frac{3 g_{3}(t, z)}{g_{2}(t, z)}}\left\{\frac{\sin \xi_{5}(t, x, z)}{\cos \xi_{5}(t, x, z) \pm 1}\right\}\right]^{2},
\end{aligned}
$$

with

$$
\begin{aligned}
& \xi_{5}(t, x, z) \\
& =k\left\{x-\int_{0}^{t}\left[\frac{-12 g_{1}^{2}(\tau, z)+25 k^{2} g_{2}(\tau, z) g_{3}(\tau, z)}{50 g_{2}(\tau, z)}\right] d \tau\right\} \\
& \quad+c .
\end{aligned}
$$

In the limit case when $m \rightarrow 1$, we have sn $\xi /(\mathrm{cn} \xi \pm \operatorname{dn} \xi) \rightarrow$ $\tanh (\xi) / 2 \operatorname{sech}(\xi)=(1 / 2) \sinh (\xi)$; thus $(24)$ becomes

$$
\begin{aligned}
& u_{6}(t, x, z) \\
& \quad=\left[-\frac{2 g_{1}(t, z)}{5 g_{2}(t, z)} \pm \frac{i k}{2} \sqrt{\frac{3 g_{3}(t, z)}{g_{2}(t, z)}} \sinh \left(\xi_{3}(t, x, z)\right)\right]^{2} .
\end{aligned}
$$

Case 3. If we take $P=1 / 4, Q=\left(1-2 m^{2}\right) / 2$, and $R=1 / 4$, then $F(\xi) \rightarrow \mathrm{ns}(\xi) \pm \mathrm{cs}(\xi)$ and

$$
\begin{aligned}
& u_{7}(t, x, z) \\
&=\left[-\frac{2 g_{1}(t, z)}{5 g_{2}(t, z)} \pm i k \sqrt{\frac{3 g_{3}(t, z)}{g_{2}(t, z)}}\right. \\
&\left.\quad \times\left\{\mathrm{ns}\left(\xi_{6}(t, x, z)\right) \pm \operatorname{cs}\left(\xi_{6}(t, x, z)\right)\right\}\right]^{2},
\end{aligned}
$$

with

$$
\begin{aligned}
& \xi_{6}(t, x, z) \\
& =k\left\{x-\int_{0}^{t}\left[\frac{-12 g_{1}^{2}(\tau, z)+25 k^{2} g_{2}(\tau, z) g_{3}(\tau, z)\left(1-2 m^{2}\right)}{50 g_{2}(\tau, z)}\right] d \tau\right\} \\
& \quad+c .
\end{aligned}
$$

In the limit case when $m \rightarrow o$, we have ns $(\xi) \pm \mathrm{cs}(\xi) \rightarrow$ $\csc (\xi) \pm \cot (\xi)$; thus (29) becomes

$$
\begin{aligned}
& u_{8}(t, x, z) \\
& =\left[-\frac{2 g_{1}(t, z)}{5 g_{2}(t, z)} \pm i k \sqrt{\frac{3 g_{3}(t, z)}{g_{2}(t, z)}}\right. \\
& \left.\quad \times\left\{\csc \left(\xi_{5}(t, x, z)\right) \pm \cot \left(\xi_{5}(t, x, z)\right)\right\}\right]^{2} .
\end{aligned}
$$

In the limit case when $m \rightarrow 1$, we have ns $(\xi) \pm$ cs $(\xi) \rightarrow$ $\operatorname{coth} \xi \pm \operatorname{csch} \xi$; thus (29) becomes

$$
u_{9}(t, x, z)
$$

$$
=\left[-\frac{2 g_{1}(t, z)}{5 g_{2}(t, z)} \pm \frac{i k}{2} \sqrt{\frac{3 g_{3}(t, z)}{g_{2}(t, z)}}\right.
$$

$$
\left.\times\left\{\operatorname{coth} \xi_{7}(t, x, z) \pm \operatorname{csch} \xi_{7}(t, x, z)\right\}\right]^{2},
$$

with

$$
\begin{aligned}
& \xi_{7}(t, x, z) \\
& =k\left\{x+\int_{0}^{t}\left[\frac{12 g_{1}^{2}(\tau, z)+25 k^{2} g_{2}(\tau, z) g_{3}(\tau, z)}{50 g_{2}(\tau, z)}\right] d \tau\right\} \\
& \quad+c .
\end{aligned}
$$

Remark that there are other solutions for (2). These solutions come from setting different values for the coefficients $P, Q$, and $R$ (see Appendices $\mathrm{A}, \mathrm{B}$, and $\mathrm{C}$ ). The above-mentioned cases are just to clarify how far our technique is applicable.

\section{White Noise Functional Solutions of (2)}

In this section, we employ the results of Section 3 by using Hermite transform to obtain exact white noise functional solutions for Wick-type stochastic Schamel KdV equations (2). The properties of exponential and trigonometric functions yield the fact that there exists a bounded open set $\mathbf{H} \subset$ $\mathbb{R}_{+} \times \mathbb{R}, \rho<\infty, \lambda>0$ such that the solution $u(t, x, z)$ of (9) and all its partial derivatives which are involved in (9) are uniformly bounded for $(t, x, z) \in \mathbf{H} \times K_{\rho}(\lambda)$, continuous with respect to $(t, x) \in \mathbf{H}$ for all $z \in K_{\rho}(\lambda)$, and analytic with respect to $z \in K_{\rho}(\lambda)$, for all $(t, x) \in \mathbf{H}$. From Theorem 4.1.1 in [47], there exists $U(t, x, z) \in(\mathcal{S})_{-1}$ such that $u(t, x, z)=$ $\widetilde{U}(t, x)(z)$ for all $(t, x, z) \in \mathbf{H} \times K_{\rho}(\lambda)$ and $U(t, x)$ solves $(2)$ in $(\mathcal{S})_{-1}$. Hence, by applying the inverse Hermite transform to the results of Section 3 , we get exact white noise functional solutions of (2) as follows. 
(i) Exact stochastic Jacobi elliptic functions solutions:

$$
\begin{gathered}
U_{1}(t, x)=\left[-\frac{2 G_{1}(t)}{5 G_{2}(t)} \pm i k \sqrt{\frac{12 G_{3}(t)}{G_{2}(t)}} \diamond \mathrm{cs}^{\diamond}\left(\Xi_{1}(t, x)\right)\right]^{\diamond 2}, \\
U_{2}(t, x)=\left[-\frac{2 G_{1}(t)}{5 G_{2}(t)} \pm i k \sqrt{\frac{3 G_{3}(t)}{G_{2}(t)}}\right. \\
\left.\qquad\left\{\frac{\mathrm{sn}^{\diamond}\left(\xi_{2}(t, x)\right)}{\mathrm{cn} \diamond\left(\Xi_{2}(t, x)\right) \pm \mathrm{dn}^{\diamond}\left(\Xi_{2}(t, x)\right)}\right\}\right]^{\diamond 2}, \\
U_{3}(t, x)=\left[-\frac{2 G_{1}(t)}{5 G_{2}(t)} \pm i k \sqrt{\frac{3 G_{3}(t)}{G_{2}(t)}}\right. \\
\left.\diamond\left\{\mathrm{ns}^{\diamond}\left(\Xi_{3}(t, x)\right) \pm c s^{\diamond}\left(\Xi_{3}(t, x)\right)\right\}\right]^{\diamond 2}
\end{gathered}
$$

with

$$
\begin{aligned}
& \Xi_{1}(t, x) \\
& =k\left\{x-\int_{0}^{t}\left[\frac{-6 G_{1}^{\diamond 2}(\tau)+25 k^{2} G_{2}(\tau) \diamond G_{3}(\tau)\left(2-m^{2}\right)}{25 G_{2}(\tau)}\right] d \tau\right\} \\
& \quad+c, \\
& \Xi_{2}(t, x) \\
& =k\left\{x-\int_{0}^{t}\left[\frac{-12 G_{1}^{\diamond 2}(\tau)+25 k^{2} G_{2}(\tau) \diamond G_{3}(\tau)\left(m^{2}+1\right)}{50 G_{2}(\tau)}\right] d \tau\right\} \\
& \quad+c, \\
& \Xi_{3}(t, x) \\
& =k\left\{x-\int_{0}^{t}\left[\frac{-12 G_{1}^{\diamond 2}(\tau)+25 k^{2} G_{2}(\tau) \diamond G_{3}(\tau)\left(1-2 m^{2}\right)}{50 G_{2}(\tau)}\right] d \tau\right\} \\
& \quad+c .
\end{aligned}
$$

(ii) Exact stochastic trigonometric solutions:

$$
\begin{aligned}
& U_{4}(t, x) \\
& =\left[-\frac{2 G_{1}(t)}{5 G_{2}(t)} \pm \sqrt{\frac{-12 k^{2} G_{3}(t)}{G_{2}(t)}} \diamond \cot ^{\diamond}\left(\Xi_{4}(t, x)\right)\right]^{\diamond 2}, \\
& U_{5}(t, x) \\
& =\left[-\frac{2 G_{1}(t)}{5 G_{2}(t)} \pm i k \sqrt{\frac{3 G_{3}(t)}{G_{2}(t)}} \diamond\left\{\frac{\sin ^{\diamond} \Xi_{5}(t, x)}{\cos ^{\diamond} \Xi_{5}(t, x) \pm 1}\right\}\right]^{\diamond 2},
\end{aligned}
$$

$U_{6}(t, x)$

$$
\begin{gathered}
=\left[-\frac{2 G_{1}(t)}{5 G_{2}(t)} \pm i k \sqrt{\frac{3 G_{3}(t)}{G_{2}(t)}}\right. \\
\left.\diamond\left\{\csc ^{\diamond}\left(\Xi_{5}(t, x)\right) \pm \cot ^{\diamond}\left(\Xi_{5}(t, x)\right)\right\}\right]^{\diamond 2},
\end{gathered}
$$

with

$$
\begin{aligned}
& \Xi_{4}(t, x) \\
& =k\left\{x-\int_{0}^{t}\left[\frac{-6 G_{1}^{\diamond 2}(\tau)+50 k^{2} G_{2}(\tau) \diamond G_{3}(\tau)}{25 G_{2}(\tau)}\right] d \tau\right\} \\
& \quad+c, \\
& \Xi_{5}(t, x) \\
& =k\left\{x-\int_{0}^{t}\left[\frac{-12 G_{1}^{\diamond 2}(\tau)+25 k^{2} G_{2}(\tau) \diamond G_{3}(\tau)}{50 G_{2}(\tau)}\right] d \tau\right\} \\
& \quad+c .
\end{aligned}
$$

(iii) Exact stochastic hyperbolic solutions:

$U_{7}(t, x)$

$$
=\left[-\frac{2 G_{1}(t)}{5 G_{2}(t)} \pm \sqrt{\frac{-12 k^{2} G_{3}(t)}{G_{2}(t)}} \diamond \operatorname{csch}^{\diamond}\left(\Xi_{6}(t, x)\right)\right]^{\diamond 2},
$$

$U_{8}(t, x)$

$$
\begin{aligned}
= & {\left[-\frac{2 G_{1}(t)}{5 G_{2}(t)} \pm \frac{i k}{2} \sqrt{\frac{3 G_{3}(t)}{G_{2}(t)}} \diamond \sinh ^{\diamond}\left(\Xi_{6}(t, x)\right)\right]^{\diamond 2}, } \\
U_{9} & (t, x) \\
& =\left[-\frac{2 G_{1}(t)}{5 G_{2}(t)} \pm \frac{i k}{2} \sqrt{\frac{3 G_{3}(t)}{G_{2}(t)}}\right.
\end{aligned}
$$

$$
\left.\diamond\left\{\operatorname{coth}^{\diamond} \Xi_{7}(t, x) \pm \operatorname{csch}^{\diamond} \Xi_{7}(t, x)\right\}\right]^{\diamond 2}
$$

with

$$
\begin{aligned}
& \Xi_{6}(t, x) \\
& =k\left\{x-\int_{0}^{t}\left[\frac{-6 G_{1}^{\diamond 2}(\tau)+25 k^{2} G_{2}(\tau) \diamond G_{3}(\tau)}{25 G_{2}(\tau)}\right] d \tau\right\} \\
& \quad+c
\end{aligned}
$$




$$
\begin{aligned}
& \Xi_{7}(t, x) \\
& =k\left\{x+\int_{0}^{t}\left[\frac{6 G_{1}^{\diamond 2}(\tau)+25 k^{2} G_{2}(\tau) \diamond G_{3}(\tau)}{50 G_{2}(\tau)}\right] d \tau\right\} \\
& \quad+c .
\end{aligned}
$$

We observe that, for different forms of $G_{1}, G_{2}$, and $G_{3}$, we can get different types of exact stochastic functional solutions of (2) from (34)-(38).

\section{Example}

It is well known that Wick version of function is usually difficult to evaluate. So, in this section, we give non-Wick version of solutions of (2). Let $W_{t}=\dot{B}_{t}$ be the Gaussian white noise, where $B_{t}$ is the Brownian motion. We have the Hermite transform [47]:

$$
\widetilde{W}_{t}(z)=\sum_{i=1}^{\infty} z_{i} \int_{0}^{t} \mu_{i}(s) d s
$$

Since

$$
\begin{aligned}
& \exp ^{\diamond}\left(B_{t}\right)=\exp \left(B_{t}-\frac{t^{2}}{2}\right), \quad \text { we have } \\
& \sin ^{\diamond}\left(B_{t}\right)=\sin \left(B_{t}-\frac{t^{2}}{2}\right), \\
& \cos ^{\diamond}\left(B_{t}\right)=\cos \left(B_{t}-\frac{t^{2}}{2}\right), \\
& \cot ^{\diamond}\left(B_{t}\right)=\cot \left(B_{t}-\frac{t^{2}}{2}\right), \\
& \csc ^{\diamond}\left(B_{t}\right)=\csc \left(B_{t}-\frac{t^{2}}{2}\right), \\
& \operatorname{coth}^{\diamond}\left(B_{t}\right)=\operatorname{coth}\left(B_{t}-\frac{t^{2}}{2}\right), \\
& \operatorname{csch}^{\diamond}\left(B_{t}\right)=\operatorname{csch}\left(B_{t}-\frac{t^{2}}{2}\right),
\end{aligned}
$$

Suppose that

$$
\begin{gathered}
G_{1}(t)=\eta_{1} G_{3}(t), \quad G_{2}(t)=\eta_{2} G_{3}(t), \\
G_{3}(t)=\sigma(t)+\eta_{3} W_{t},
\end{gathered}
$$

where $\eta_{1}, \eta_{2}$, and $\eta_{3}$ are arbitrary constants and $\sigma(t)$ is integrable or bounded measurable function on $\mathbb{R}_{+}$. Therefore, for
$G_{1}(t) G_{2}(t) G_{3}(t) \neq 0$, exact white noise functional solutions of (2) are as follows:

$$
\begin{aligned}
& U_{10}(t, x)=\left[-\frac{2 \eta_{1}}{5 \eta_{2}} \pm i k \sqrt{\frac{12}{\eta_{2}}} \cot \Pi_{1}(t, x)\right]^{2}, \\
& U_{11}(t, x)=\left[-\frac{2 \eta_{1}}{5 \eta_{2}} \pm i k \sqrt{\frac{3}{\eta_{2}}}\left\{\frac{\sin \Pi_{2}(t, x)}{\cos \Pi_{2}(t, x) \pm 1}\right\}\right]^{2}, \\
& U_{12}(t, x) \\
& =\left[-\frac{2 \eta_{1}}{5 \eta_{2}} \pm i k \sqrt{\frac{3}{\eta_{2}}}\left\{\csc \Pi_{2}(t, x) \pm \cot \Pi_{2}(t, x)\right\}\right]^{2},
\end{aligned}
$$

with

$$
\begin{aligned}
& \Pi_{1}(t, x)=k\left[x-\left(\frac{-6 \eta_{1}^{2}+50 k^{2} \eta_{2}}{25 \eta_{2}}\right)\right. \\
& \left.\times\left\{\int_{0}^{t} \sigma(\tau) d \tau+\eta_{3}\left[B_{t}-\frac{t^{2}}{2}\right]\right\}\right]+c, \\
& \Pi_{2}(t, x)=k\left[x-\left(\frac{-12 \eta_{1}^{2}+25 k^{2} \eta_{2}}{50 \eta_{2}}\right)\right. \\
& \left.\times\left\{\int_{0}^{t} \sigma(\tau) d \tau+\eta_{3}\left[B_{t}-\frac{t^{2}}{2}\right]\right\}\right]+c, \\
& U_{13}(t, x)=\left[-\frac{2 \eta_{1}}{5 \eta_{2}} \pm i k \sqrt{\frac{12}{\eta_{2}}} \operatorname{csch} \Pi_{3}(t, x)\right]^{2} \text {, } \\
& U_{14}(t, x)=\left[-\frac{2 \eta_{1}}{5 \eta_{2}} \pm \frac{i k}{2} \sqrt{\frac{3}{\eta_{2}}} \sinh \Pi_{3}(t, x)\right]^{2}, \\
& U_{15}(t, x) \\
& =\left[-\frac{2 \eta_{1}}{5 \eta_{2}} \pm \frac{i k}{2} \sqrt{\frac{3}{\eta_{2}}}\left\{\operatorname{coth} \Pi_{4}(t, x) \pm \operatorname{csch} \Pi_{4}(t, x)\right\}\right]^{2},
\end{aligned}
$$

with

$$
\begin{aligned}
& \Pi_{3}(t, x) \\
& =k\left[x-\frac{-6 \eta_{1}^{2}+25 k^{2} \eta_{2}}{25 \eta_{2}}\left\{\int_{0}^{t} \sigma(\tau) d \tau+\eta_{3}\left[B_{t}-\frac{t^{2}}{2}\right]\right\}\right] \\
& \quad+c
\end{aligned}
$$

$\Pi_{4}(t, x)$

$=k\left[x+\frac{12 \eta_{1}^{2}+25 k^{2} \eta_{2}}{50 \eta_{2}}\left\{\int_{0}^{t} \sigma(\tau) d \tau+\eta_{3}\left[B_{t}-\frac{t^{2}}{2}\right]\right\}\right]$

$+c$. 
TABLE 1

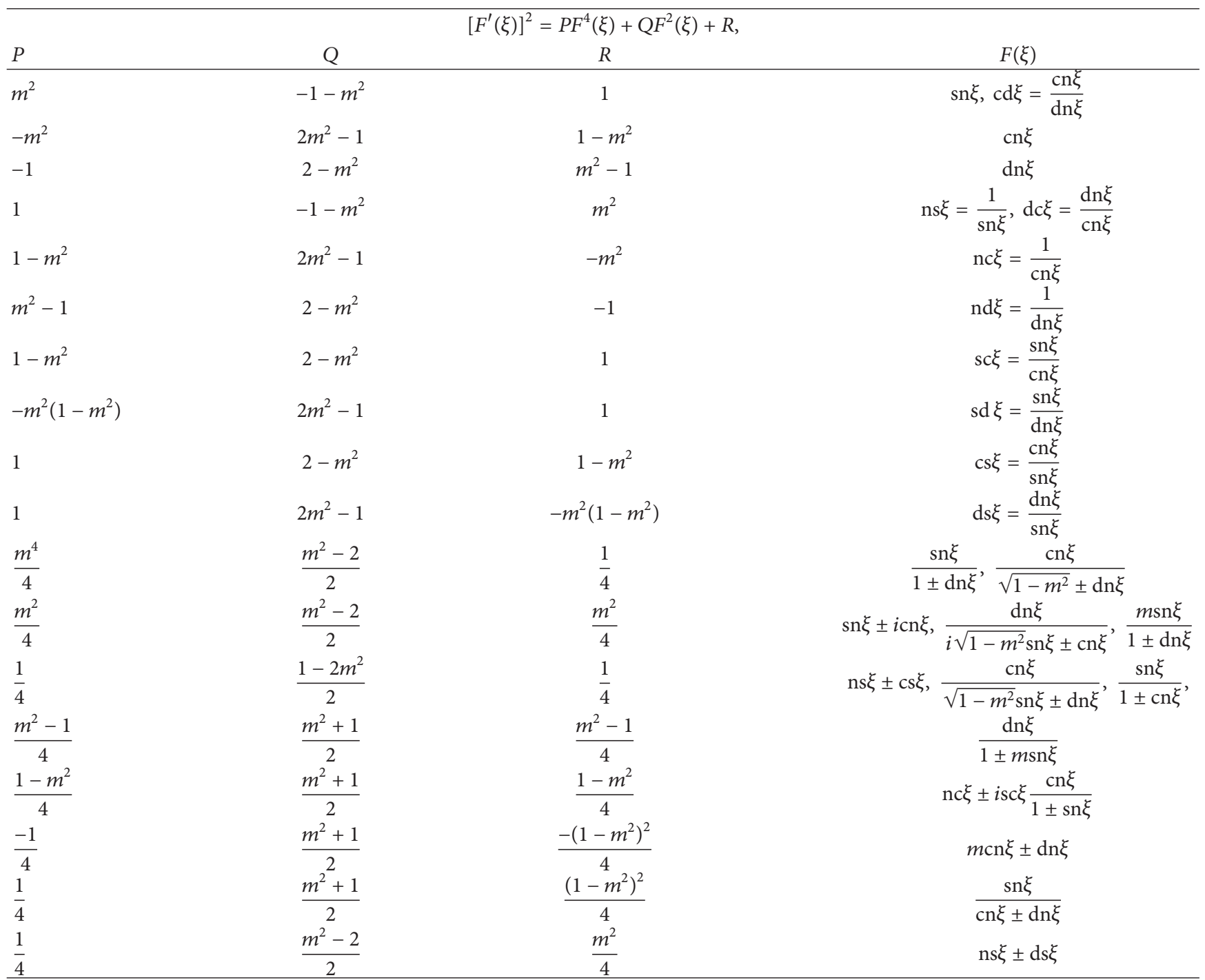

\section{Summary and Discussion}

We have discussed the solutions of SPDEs driven by Gaussian white noise. There is a unitary mapping between the Gaussian white noise space and the Poisson white noise space. This connection was given by Benth and Gjerde [48]. From [47, section 4.9] and by the aid of the connection, we can derive some stochastic exact soliton solutions, which are Poisson white noise functions in (2). In this paper, using Hermite transformation, white noise theory, and F-expansion method, we study the white noise functional solutions for Wick-type stochastic Schamel KdV equations. This paper shows that F-expansion method is sufficient to solve the stochastic nonlinear equations in mathematical physics. The method which we have proposed in this paper is standard, direct, and computerized method, which allows us to do complicated and tedious algebraic calculation. It is shown that the algorithm can be also applied to other nonlinear SPDEs in mathematical physics such as modified HirotaSatsuma coupled KdV, KdV-Burgers, modified KdV Burgers,
Sawada-Kotera, and Zhiber-Shabat equations and BenjaminBona-Mahony (BBM) equations. Since (2) has other solutions of Jacobi elliptic functions, trigonometric functions, and hyperbolic functions if we select other values of $P, Q$, and $R$ (see Appendices $\mathrm{A}, \mathrm{B}$, and $\mathrm{C}$ ), there are many other exact traveling wave solutions for Wick-type stochastic Schamel $\mathrm{KdV}$ equations.

\section{Appendices}

\section{A.}

The Jacobi elliptic functions degenerate into trigonometric functions when $m \rightarrow 0$ :

$$
\begin{aligned}
& \operatorname{sn} \xi \longrightarrow \sin \xi, \quad \operatorname{cn} \xi \longrightarrow \cos \xi, \quad \operatorname{dn} \xi \longrightarrow 1, \\
& \operatorname{sc} \xi \longrightarrow \tan \xi, \operatorname{sd} \longrightarrow \sin \xi, \quad \operatorname{cd} \xi \longrightarrow \cos \xi, \\
& \mathrm{ns} \xi \longrightarrow \csc \xi, \quad \text { nc } \xi \longrightarrow \sec \xi, \quad \text { nd } \xi \longrightarrow 1,
\end{aligned}
$$




$$
\begin{aligned}
& \operatorname{cs} \xi \longrightarrow \cot \xi, \\
& \text { ds } \xi \longrightarrow \csc \xi, \quad \text { dc } \xi \longrightarrow \sec \xi .
\end{aligned}
$$

B.

The Jacobi elliptic functions degenerate into hyperbolic functions when $m \rightarrow 1:$

$$
\begin{aligned}
& \operatorname{sn} \xi \longrightarrow \tan \xi, \quad \operatorname{cn} \xi \longrightarrow \operatorname{sech} \xi, \quad \operatorname{dn} \xi \longrightarrow \operatorname{sech} \xi, \\
& \operatorname{sc} \xi \longrightarrow \sinh \xi \\
& \operatorname{sd} \xi \longrightarrow \sinh \xi, \quad \mathrm{cd} \xi \longrightarrow 1, \\
& \text { ns } \xi \longrightarrow \operatorname{coth} \xi, \quad \text { nc } \xi \longrightarrow \cosh \xi, \quad \text { nd } \xi \longrightarrow \cosh \text {, } \\
& \operatorname{cs} \xi \longrightarrow \operatorname{csch} \xi \\
& \text { ds } \xi \longrightarrow \operatorname{csch} \xi, \quad \text { dc } \xi \longrightarrow 1 \text {. }
\end{aligned}
$$

\section{C.}

The ODE and Jacobi elliptic functions: for relation between values of $P, Q$, and $R$ and corresponding $F(\xi)$ in ODE, see Table 1.

\section{Conflict of Interests}

The authors declare that there is no conflict of interests regarding the publication of this paper.

\section{References}

[1] A. H. Khater, M. M. Hassan, and R. S. Temsah, "Exact solutions with Jacobi elliptic functions of two nonlinear models for ionacoustic plasma waves," Journal of the Physical Society of Japan, vol. 74, no. 5, pp. 1431-1435, 2005.

[2] H. Schamel, "A modified Korteweg de Vries equation for ion acoustic waves due to resonant electrons," Journal of Plasma Physics, vol. 9, pp. 377-387, 1973.

[3] M. Wadati, "Stochastic Korteweg-de Vries equation," Journal of the Physical Society of Japan, vol. 52, no. 8, pp. 2642-2648, 1983.

[4] M. Wadati and Y. Akutsu, "Stochastic Korteweg-de Vries equation with and without damping," Journal of the Physical Society of Japan, vol. 53, no. 10, pp. 3342-3350, 1984.

[5] A. de Bouard and A. Debussche, "On the stochastic Kortewegde Vries equation," Journal of Functional Analysis, vol. 154, no. 1, pp. 215-251, 1998.

[6] A. de Bouard, A. Debussche, and Y. Tsutsumi, "White noise driven Korteweg-de Vries equation," Journal of Functional Analysis, vol. 169, no. 2, pp. 532-558, 1999.

[7] A. Debussche and J. Printems, "Numerical simulation of the stochastic Korteweg-de Vries equation," Physica D, vol. 134, no. 2, pp. 200-226, 1999.

[8] A. Debussche and J. Printems, "Effect of a localized random forcing term on the Korteweg-de Vries equation," Journal of Computational Analysis and Applications, vol. 3, no. 3, pp. 183206, 2001.
[9] V. V. Konotop and L. Vazquez, Nonlinear Random Waves, World Scientific Publishing, Singapore, 1994.

[10] Y. Xie, "Exact solutions for stochastic KdV equations," Physics Letters A, vol. 310, no. 2-3, pp. 161-167, 2003.

[11] Y. C. Xie, "Exact solutions of the Wick-type stochastic Kadomtsev-Petviashvili equations," Chaos, Solitons and Fractals, vol. 21, no. 2, pp. 473-480, 2004.

[12] Y. Xie, "Positonic solutions for Wick-type stochastic KdV equations," Chaos, Solitons and Fractals, vol. 20, no. 2, pp. 337342, 2004.

[13] Y. Xie, "An auto-Bäcklund transformation and exact solutions for Wick-type stochastic generalized KdV equations," Journal of Physics A: Mathematical and General, vol. 37, no. 19, pp. 52295236, 2004.

[14] Y. C. Xie, "Exact solutions for stochastic KdV equations," Physics Letters A, vol. 310, no. 2-3, pp. 161-167, 2003.

[15] H. A. Ghany, "Exact solutions for stochastic generalized HirotaSatsuma coupled KdV equations," Chinese Journal of Physics, vol. 49, no. 4, pp. 926-940, 2011.

[16] H. A. Ghany and A. Fathallah, "Exact solutions for KDVburger equations with an application of white-noise analysis," International Journal of Pure and Applied Mathematics, vol. 78, no. 1, pp. 17-27, 2012.

[17] H. A. Ghany and A.-A. Hyder, "White noise functional solutions for the Wick-type two-dimensional stochastic ZakharovKuznetsov equations," International Review of Physics, vol. 6, no. 2, pp. 153-157, 2012.

[18] H. A. Ghany and A.-A. Hyder, "Exact solutions for the wicktype stochastic time-fractional KdV equations," Kuwait Journal of Science, vol. 41, no. 1, pp. 75-84, 2014.

[19] H. A. Ghany and A. Hyder, "Abundant solutions of Wick-type stochastic fractional 2D KdV equations," Chinese Physics B, vol. 23, no. 6, Article ID 060503, 2014.

[20] H. A. Ghany and M. S. Mohammed, "White noise functional solutions for Wick-type stochastic fractional KdV-BurgersKuramoto equations," Chinese Journal of Physics, vol. 50, no. 4, pp. 619-627, 2012.

[21] H. A. Ghany, A. S. Okb El Bab, A. M. Zabel, and A.-A. Hyder, "The fractional coupled $\mathrm{KdV}$ equations: exact solutions and white noise functional approach," Chinese Physics B, vol. 22, no. 8, Article ID 080501, 2013.

[22] S. Abbasbandy, "Approximate solution for the nonlinear model of diffusion and reaction in porous catalysts by means of the homotopy analysis method," Chemical Engineering Journal, vol. 136, no. 2-3, pp. 144-150, 2008.

[23] M. Dehghan, J. M. Heris, and A. Saadatmandi, "Application of semi-analytic methods for the Fitzhugh-Nagumo equation, which models the transmission of nerve impulses," Mathematical Methods in the Applied Sciences, vol. 33, no. 11, pp. 1384-1398, 2010.

[24] M. Dehghan and M. Tatari, "Identifying an unknown function in a parabolic equation with overspecified data via He's variational iteration method," Chaos, Solitons \& Fractals, vol. 36, no. 1, pp. 157-166, 2008.

[25] E. Fan, "Extended tanh-function method and its applications to nonlinear equations," Physics Letters A, vol. 277, no. 4-5, pp. 212-218, 2000.

[26] A. M. Wazwaz, "The tanh method for generalized forms of nonlinear heat conduction and Burgers-Fisher equations," Applied Mathematics and Computation, vol. 169, no. 1, pp. 321338, 2005. 
[27] S. Zhang and T.-C. Xia, "Symbolic computation and new families of exact non-travelling wave solutions of $(2+1)$ dimensional Broer-Kaup equations," Communications in Theoretical Physics, vol. 45, no. 6, pp. 985-990, 2006.

[28] D. D. Ganji and A. Sadighi, "Application of He's homotopyperturbation method to nonlinear coupled systems of reactiondiffusion equations," International Journal of Nonlinear Sciences and Numerical Simulation, vol. 7, no. 4, pp. 411-418, 2006.

[29] F. Shakeri and M. Dehghan, "Solution of delay differential equations via a homotopy perturbation method," Mathematical and Computer Modelling, vol. 48, no. 3-4, pp. 486-498, 2008.

[30] X. Ma, L. Wei, and Z. Guo, "He's homotopy perturbation method to periodic solutions of nonlinear Jerk equations," Journal of Sound and Vibration, vol. 314, no. 1-2, pp. 217-227, 2008.

[31] A.-M. Wazwaz, "The tanh-coth method for new compactons and solitons solutions for the $K(n ; n)$ and the $K(n+1, n+1)$ equations," Applied Mathematics and Computation, vol. 188, no. 2, pp. 1930-1940, 2007.

[32] A.-M. Wazwaz, "Travelling wave solutions for combined and double combined sine-cosine-Gordon equations by the variable separated ODE method," Applied Mathematics and Computation, vol. 177, no. 2, pp. 755-760, 2006.

[33] H. A. Ghany and M. Zakarya, "Generalized solutions of Wicktype stochastic KdV-burgers equations using exp-function method," International Review of Physics, vol. 8, no. 2, 2014.

[34] J.-H. He and X.-H. Wu, "Exp-function method for nonlinear wave equations," Chaos, Solitons \& Fractals, vol. 30, no. 3, pp. 700-708, 2006.

[35] J.-H. He and M. A. Abdou, "New periodic solutions for nonlinear evolution equations using Exp-function method," Chaos, Solitons \& Fractals, vol. 34, no. 5, pp. 1421-1429, 2007.

[36] X.-H. Wu and J.-H. He, "EXP-function method and its application to nonlinear equations," Chaos, Solitons \& Fractals, vol. 38, no. 3, pp. 903-910, 2008.

[37] S.-D. Zhu, "Exp-function method for the Hybrid-Lattice system," International Journal of Nonlinear Sciences and Numerical Simulation, vol. 8, no. 3, pp. 461-464, 2007.

[38] S.-D. Zhu, "Exp-function method for the discrete mKdV lattice," International Journal of Nonlinear Sciences and Numerical Simulation, vol. 8, no. 3, pp. 465-468, 2007.

[39] Z. Fu, S. Liu, and Q. Zhao, "New Jacobi elliptic function expansion and new periodic solutions of nonlinear wave equations," Physics Letters A, vol. 290, no. 1-2, pp. 72-76, 2001.

[40] S. K. Liu, Z. T. Fu, S. D. Liu, and Q. Zhao, "Jacobi elliptic function expansion method and periodic wave solutions of nonlinear wave equations," Physics Letters A, vol. 289, no. 1-2, pp. 69-74, 2001.

[41] J. Liu, L. Yang, and K. Yang, "Nonlinear transform and Jacobi elliptic function solutions of nonlinear equations," Chaos, Solitons and Fractals, vol. 20, no. 5, pp. 1157-1164, 2004.

[42] E. J. Parkes, B. R. Duffy, and P. C. Abbott, "The Jacobi elliptic-function method for finding periodic-wave solutions to nonlinear evolution equations," Physics Letters A, vol. 295, no. 5-6, pp. 280-286, 2002.

[43] Y. Zhou, M. Wang, and Y. Wang, "Periodic wave solutions to a coupled KdV equations with variable coefficients," Physics Letters A, vol. 308, no. 1, pp. 31-36, 2003.

[44] S. Zhang and T. Xia, "A generalized F-expansion method with symbolic computation exactly solving Broer-Kaup equations," Applied Mathematics and Computation, vol. 189, no. 1, pp. 836843, 2007.
[45] S. Zhang and T. Xia, "A generalized F-expansion method and new exact solutions of Konopelchenko-Dubrovsky equations," Applied Mathematics and Computation, vol. 183, no. 2, pp. 11901200, 2006.

[46] S. Zhang and T. Xia, "An improved generalized F-expansion method and its application to the $(2+1)$-dimensional KdV equations," Communications in Nonlinear Science and Numerical Simulation, vol. 13, no. 7, pp. 1294-1301, 2008.

[47] H. Holden, B. Øksendal, J. Ubøe, and T. Zhang, Stochastic Partial Differential Equations, Birkhäuser, Basel, Switzerland, 1996.

[48] F. E. Benth and J. Gjerde, "A remark on the equivalence between Poisson and Gaussian stochastic partial differential equations," Potential Analysis, vol. 8, no. 2, pp. 179-193, 1998. 

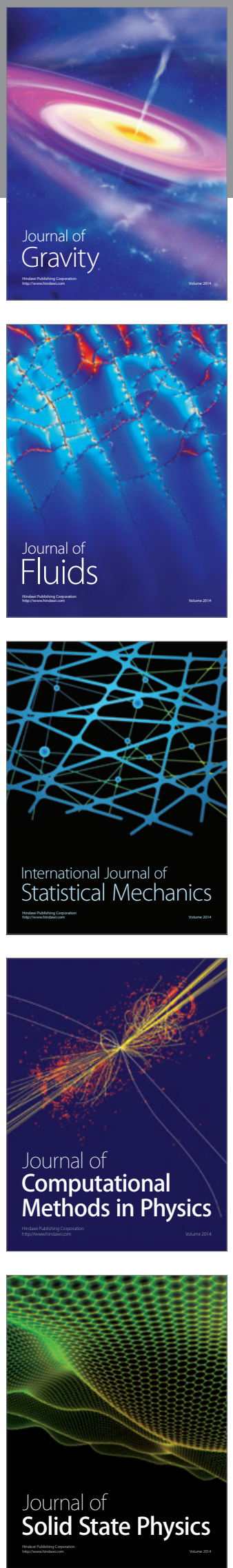

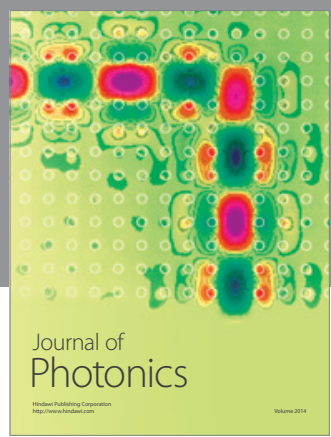

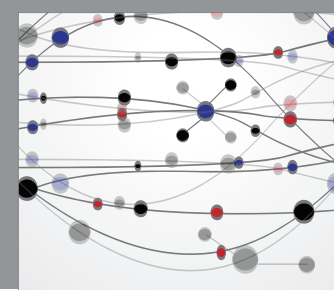

The Scientific World Journal

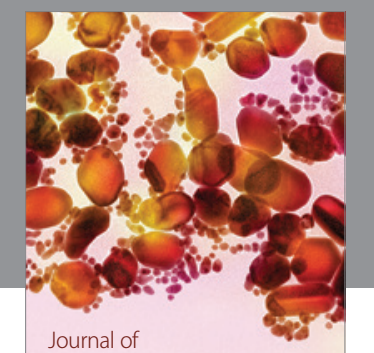

Soft Matter
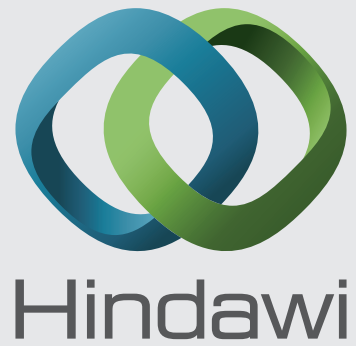

Submit your manuscripts at

http://www.hindawi.com
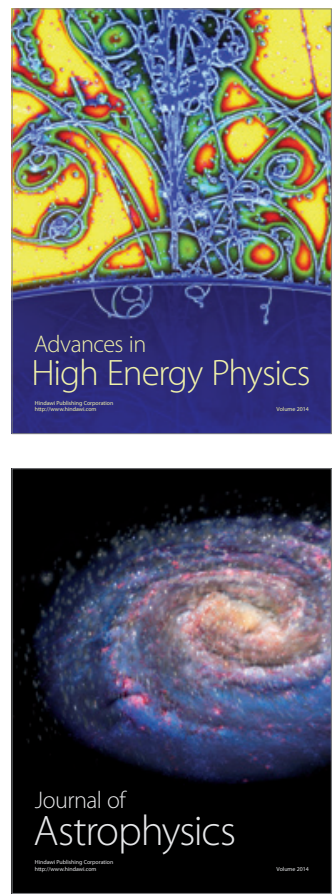
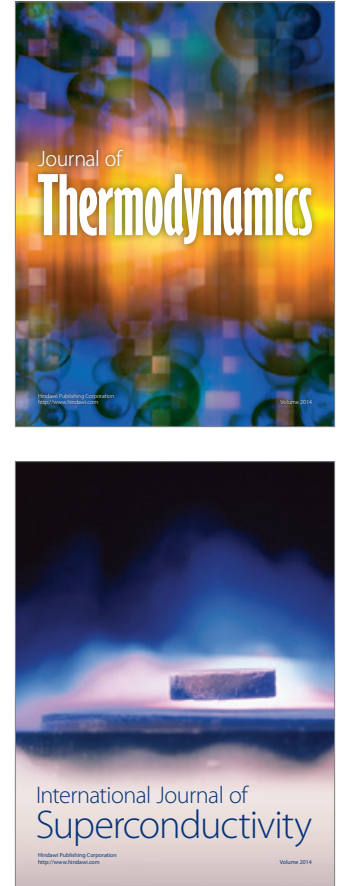
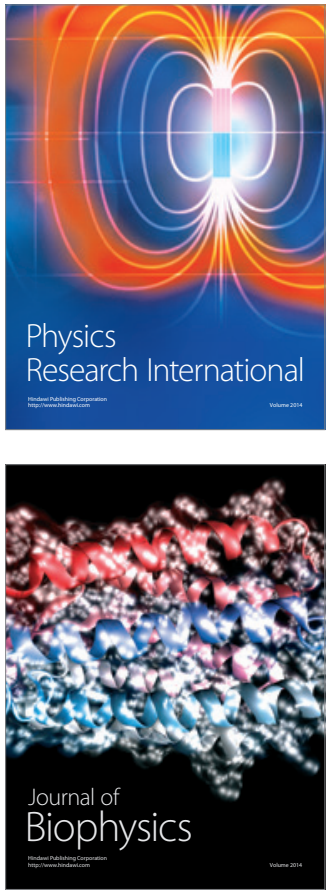
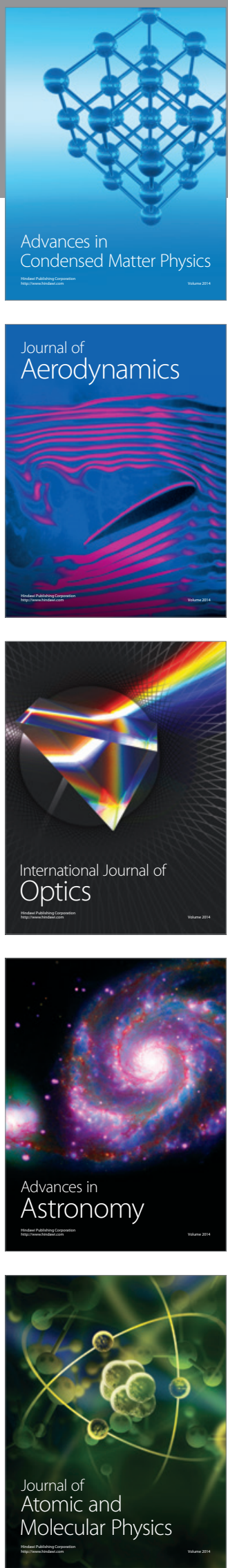